\title{
ON THE COEFFICIENT PROBLEM FOR THE SCHWARZIAN DERIVATIVE
}

\author{
Stephen M. Zemyan \\ The Pennsylvania State University, Department of Mathematics, Mont Alto Campus \\ Campus Drive, Mont Alto, PA 17237-9799, U.S.A.
}

\begin{abstract}
For $f \in S$, we study the Schwarzian coefficients $s_{n}$ defined by $\{f, z\}=\Sigma s_{n} z^{n}$. The problem of determining the value $\max _{S} \operatorname{Re}\left\{s_{N}\right\}$ and the functions for which this maximum is attained is approached using variational methods. Necessary coefficient inequalities are obtained as an application of the spire variation, and a quadratic differential equation involving Faber polynomials is derived using Schiffer's boundary variation. It is also shown that any zero of the associated quadratic differential on the omitted set is simple.
\end{abstract}

\section{Introduction}

Within the theory of functions of a complex variable, the Schwarzian derivative appears in connection with univalence criteria [3, p. 258], conformal mapping [7, p. 199], homeomorphic and quasiconformal extensions and the growing theory of Teichmüller spaces [5]. Indeed, Bernardi [1] lists more than one hundred references to books and papers which are concerned with the Schwarzian derivative. A concise introduction to this vast and important theory is given by Lehto [5, p. $51 \mathrm{ff}]$.

If a function $f(z)$ is analytic and locally univalent in the domain $D$, then $f^{\prime}(z) \neq 0$ there, and we may define the Schwarzian derivative of $f$ in $D$ by the relation

$$
\{f, z\}=\left(\frac{f^{\prime \prime}(z)}{f^{\prime}(z)}\right)^{\prime}-\frac{1}{2}\left(\frac{f^{\prime \prime}(z)}{f^{\prime}(z)}\right)^{2}
$$

Consequently, we may define Schwarzian coefficients for $f(z)$ near a finite point $z_{0}$ by means of an expansion the form

$$
\{f, z\}=\sum s_{n}\left(f ; z_{0}\right)\left(z-z_{0}\right)^{n} .
$$

Rather than considering these coefficients in full generality, we restrict our attention here to the Schwarzian coefficients of univalent functions belonging to two well-known classes.

1991 Mathematics Subject Classification: Primary 30C50; Secondary 30C70. 
Let $S$ denote the class of functions $f(z)=z+a_{2} z^{2}+a_{3} z^{3}+\cdots$ which are analytic and univalent in the unit disk $U=\{z:|z|<1\}$. We define the Schwarzian coefficients $s_{n}$ of $f$ (near $z_{0}=0$ ) to be the coefficients in the Taylor expansion

$$
\{f, z\}=\sum_{n=0}^{\infty} s_{n} z^{n} .
$$

If necessary, we shall write $s_{n}(f)$ in order to avoid confusion.

In this paper, we consider the problem of determining the value

$$
\hat{s}_{N}=\max _{f \in S}\left|s_{N}(f)\right| \quad(N \geq 0)
$$

and the corresponding extremal functions. Equivalently, we may consider the problem of determining

$$
\hat{s}_{N}=\max _{f \in S} \operatorname{Re}\left\{s_{N}(f)\right\}
$$

since the set $\left\{s_{n}(f): f \in S\right\}$ is a closed disk centered at the origin. Indeed, if $f \in S$, then $f_{\eta}(z)=f(\eta z) / \eta \in S$ and $s_{n}\left(f_{\eta}\right)=\eta^{n+2} s_{n}(f)$, whenever $|\eta| \leq 1$.

Another formulation of this problem is possible. Let $\Sigma$ denote the class of functions $g(z)=z+b_{0}+b_{1} z^{-1}+b_{2} z^{-2}+\cdots$ which are meromorphic and univalent in $\Delta=\{z:|z|>1\}$. We define the Schwarzian coefficients $\sigma_{n}$ of $g$ (near $z_{0}=\infty$ ) to be the coefficients in the Laurent expansion

$$
\{g, z\}=z^{-4} \sum_{n=0}^{\infty} \sigma_{n} z^{-n} .
$$

In an analogous manner, we could consider the problem of determining the value

$$
\hat{\sigma}_{N}=\max _{g \in \Sigma} \operatorname{Re}\left\{\sigma_{N}(g)\right\}
$$

and the corresponding extremal functions. However, it is easy to see that problems (3) and (5) are actually equivalent, by utilizing an inversion. If $f \in S$, then $g(z)=1 / f(1 / z) \in \Sigma$; conversely, if $g \in \Sigma$ and $c$ is chosen so that $g(z)+c \neq 0$, then there exists an $f_{c} \in S$ such that $g(z)+c=1 / f_{c}(1 / z)$. The Schwarzian composition law [3, p. 259]

$$
\{f \circ w, z\}=\{f, w(z)\}\left(w^{\prime}(z)\right)^{2}+\{w, z\}
$$

implies that $\{g, z\}=z^{-4}\{f, 1 / z\}$. Hence, a comparison of (2) and (4) shows that $s_{N}(f)=\sigma_{N}(g)$, for any $f \in S$, and that $\sigma_{N}(g)=\sigma_{N}(g+c)=s_{N}\left(f_{c}\right)$, for any 
$g \in \Sigma$. It follows that $\hat{\sigma}_{N}=\hat{s}_{N}$ for any $N \geq 0$, and that the corresponding extremal functions are related by inversion and translation. In theory then, we need only consider one formulation or the other. However, in certain respects, it may be computationally more advantageous to favor one formulation over the other. Indeed, we shall have occasion to establish results with reference to both classes.

Some partial results are known. In [12], the author has shown that $\hat{s}_{0}=$ $6, \hat{s}_{1}=16$ and $\hat{s}_{2}=30\left(1+2 e^{-34 / 3}\right)$. The corresponding extremal functions are quite well-known, and will be described after our general discussion to follow of the associated quadratic differential equation. The author also establishes the uniform bound $\hat{s}_{N} \leq \frac{3}{4} \pi(N+2)^{2}, N \geq 0$, in addition to other inequalities and results which will be recalled as needed.

Most of our further results, including a great many necessary conditions for the extremal functions and their coefficients, may be obtained through the use of the following extremely useful

Variational Lemma. Let $f \in S(\Sigma)$.

(a) If $f^{*}(z)=f(z)+\varepsilon h(z)+\varepsilon^{2} r(z)+o\left(\varepsilon^{2}\right) \in S(\Sigma)$, then

$$
\left\{f^{*}, z\right\}=\{f, z\}+\varepsilon l(h ; f)+\varepsilon^{2} m(h, r ; f)+o\left(\varepsilon^{2}\right)
$$

where

$$
l(h ; f)=\left(\frac{h^{\prime}}{f^{\prime}}\right)^{\prime \prime}-\frac{f^{\prime \prime}}{f^{\prime}} \cdot\left(\frac{h^{\prime}}{f^{\prime}}\right)^{\prime}
$$

and

$$
m(h, r ; f)=l(r ; f)+\left(\frac{h^{\prime}}{f^{\prime}}\right)^{2}(\{f, z\}-\{h, z\})-3\left(\left(\frac{h^{\prime}}{f^{\prime}}\right)^{\prime}\right)^{2}
$$

(b) If $f^{*}(z)=f(z)+\varrho^{2} h(z)+\varrho^{3} r(z)+o\left(\varrho^{3}\right) \in S(\Sigma)$, then

$$
\left\{f^{*}, z\right\}=\{f, z\}+\varrho^{2} l(h ; f)+\varrho^{3} l(r ; f)+o\left(\varrho^{3}\right) .
$$

This lemma is established through direct computation. It is worthwhile to note here that for every integer $n$, we have

$$
l\left(f^{n} ; f\right)=n(n-1)(n-2) f^{n-3}\left(f^{\prime}\right)^{2}
$$

and that

$$
l\left(z^{n} f^{\prime} ; f\right)=z^{n}\{f, z\}^{\prime}+2 n z^{n-1}\{f, z\}+n(n-1)(n-2) z^{n-3} .
$$


In this paper, we shall employ general variational methods in the usual way to obtain necessary conditions for extremal functions, their omitted sets, and their coefficients. The technique typically produces inequalities, equations and geometrical restrictions of interest.

If $f \in S$ is extremal, then certainly

$$
\operatorname{Re} s_{N}\left(f^{*}\right) \leq \operatorname{Re} s_{N}(f)
$$

for every competitor $f^{*} \in S$. If the linear functional $L_{N}(h)$ denotes the coefficient of $z^{N}$ in the series expansion of $h$, then $s_{N}(f)=L_{N}(\{f, z\})$. In view of the Variational Lemma (a), (12) implies that

$$
\operatorname{Re} L_{N}(l(h ; f)) \leq 0
$$

as $\varepsilon \rightarrow 0$. Furthermore, if equality holds in (13), then we may also deduce that

$$
\operatorname{Re} L_{N}(m(h, r ; f)) \leq 0 .
$$

Thus, our results depend upon the possible choices for $h$ and $r$. Analogous inequalities hold for extremal functions in $\Sigma$.

In Chapter 2, we use the spire variation to show in Proposition 1 that the Schwarzian coefficients of an extremal function must satisfy certain restrictive inequalities.

In Chapter 3, we use Schiffer's powerful method of boundary variation to produce in Theorem 1 a quadratic differential equation satisfied by any extremal function. Theorem 2 establishes the fact that a zero of the associated quadratic differential $Q_{N}(w) d w^{2}$ which occurs on the omitted set $\Gamma_{f}$ must be simple, using an argument devised by Leung and Schober, which combines Schiffer's truncation method with Bombieri's second variation. We close with a short discussion of the extremal functions when $N=0,1$, or 2 .

\section{The spire variation}

We now introduce an elementary variation which will yield interesting coefficient inequalities for extremal functions.

The Koebe function, $k_{\eta}(z)=z /(1+\eta z)^{2},|\eta|=1$, belongs to the class $S$ and maps $U$ onto the plane with a radial slit from $k_{\eta}(\bar{\eta})=\bar{\eta} / 4$ to $\infty$. For $\varepsilon \in(0,1)$, the composition $s_{\varepsilon, \eta}(z)=k_{\eta}^{-1}\left((1-\varepsilon) k_{\eta}(z)\right)$ maps $U$ onto $U$ less a short linear, radial slit emanating from $\bar{\eta}$.

Now, if $f \in S$, then $f_{\varepsilon, \eta}(z)=f\left(s_{\varepsilon, \eta}(z)\right) / s_{\varepsilon, \eta}^{\prime}(0) \in S$, and $f_{\varepsilon, \eta}$ is known as the spire variation of $f$. In order to utilize the Variational Lemma, we first expand $s_{\varepsilon, \eta}$ and then $f_{\varepsilon, \eta}$ in powers of $\varepsilon, \varepsilon$ small. Indeed, a straightforward application of Taylor's Theorem yields

$$
s_{\varepsilon, \eta}(z)=z-\varepsilon z\left(\frac{1+\eta z}{1-\eta z}\right)+\varepsilon^{2} z\left(\frac{1+\eta z}{1-\eta z}\right)\left(\frac{1}{(1-\eta z)^{2}}-1\right)+o\left(\varepsilon^{2}\right)
$$


and

$$
f_{\varepsilon, \eta}(z)=f(z)+\varepsilon h_{\eta}(z)+\varepsilon^{2} r_{\eta}(z)+o\left(\varepsilon^{2}\right)
$$

as $\varepsilon \rightarrow 0$, where

$$
h_{\eta}(z)=f(z)-z f^{\prime}(z)\left(\frac{1+\eta z}{1-\eta z}\right)
$$

and

$$
r_{\eta}(z)=f(z)-z f^{\prime}(z)\left(\frac{1+\eta z}{1-\eta z}\right)\left(2-\frac{1}{(1-\eta z)^{2}}\right)+\frac{1}{2} z^{2} f^{\prime \prime}(z)\left(\frac{1+\eta z}{1-\eta z}\right)^{2} .
$$

Consequently, from the Variational Lemma, we obtain for $\varepsilon \in(0,1)$ and $|\eta|=1$,

$$
\left\{f_{\varepsilon, \eta}, z\right\}=\{f, z\}+\varepsilon l\left(h_{\eta} ; f\right)+\varepsilon^{2} m\left(h_{\eta}, r_{\eta} ; f\right)+o\left(\varepsilon^{2}\right),
$$

where $l$ and $m$ are defined by (8) and (9).

Now let $\mu$ be an arbitrary probability measure on $|\eta|=1$, and set

$$
p(z)=\int_{|\eta|=1} \frac{1+\eta z}{1-\eta z} d \mu(\eta) .
$$

Then, by the Herglotz Representation Theorem, $p(0)=1$ and $\operatorname{Re} p(z)>0$ for all $z \in U[11$, p. 4]. Furthermore, every analytic function of positive real part with $p(0)=1$ may be represented in this manner. We denote the class of all such $p(z)$ by $P$.

After integrating (15) over $|\eta|=1$, and noting the continuity of the functionals $L_{N}, l$ and $m$, we obtain

$$
\int_{|\eta|=1} s_{N}\left(f_{\varepsilon, \eta}\right) d \mu(\eta)=s_{N}(f)+\varepsilon L_{N}\left(l\left(h_{p} ; f\right)\right)+\varepsilon^{2} L_{N}\left(m\left(h_{p}, r_{p} ; f\right)\right)+o\left(\varepsilon^{2}\right)
$$

where

$$
\begin{aligned}
h_{p}(z)= & f(z)-z f^{\prime}(z) p(z) \\
r_{p}(z)= & f(z)+\frac{1}{2} z f^{\prime}(z)\left(1-3 p(z)+3 z p^{\prime}(z)+z^{2} p^{\prime \prime}(z)\right) \\
& +\frac{1}{2} z^{2} f^{\prime \prime}(z)\left(1+2 z p^{\prime}(z)\right) .
\end{aligned}
$$

Now, if $f$ is an extremal function for the problem (3), then $\operatorname{Re} s_{N}\left(f_{\varepsilon, \eta}\right) \leq \hat{s}_{N}$ for every choice of $\varepsilon$ and $\eta$. Since the real part of the left-hand side of (16) is an average of these lesser values, we conclude as $\varepsilon \rightarrow 0$ that

$$
\operatorname{Re} L_{N}\left(l\left(h_{p} ; f\right)\right) \leq 0
$$


for every $p \in P$; however, since $l(f ; f)=0$, this condition may be rewritten as

$$
\operatorname{Re} L_{N}\left(l\left(z f^{\prime} p ; f\right)\right) \geq 0 \quad(p \in P) .
$$

Furthermore, if equality holds in (18), then we may also conclude as $\varepsilon \rightarrow 0$ that

$$
\operatorname{Re} L_{N}\left(m\left(h_{p}, r_{p} ; f\right)\right) \leq 0 \quad(p \in P) .
$$

As an application of these observations, we provide necessary conditions satisfied by the early coefficients of an extremal function which will be significant in the next section.

Proposition 1. Let $f \in S$ and let $s_{n}(n=0,1, \ldots)$ denote its Schwarzian coefficients. Suppose that $f$ is extremal for the problem

$$
\hat{s}_{N}=\max _{f \in S} \operatorname{Re}\left\{s_{N}(f)\right\} .
$$

(a) For every $\zeta \in U$ and every $n=0, \ldots, N$, we have

$$
\operatorname{Re}\left\{\zeta s_{n}\right\} \leq \frac{N+2}{2 N+2-n} \hat{s}_{N}
$$

(b) For every $\eta,|\eta|=1$, we have

$$
\operatorname{Re}\left\{2(N+1)(N+2)(N+3) \eta^{N+2}+(N+2) s_{N}+2 \sum_{j=0}^{N-1}(2 N+2-j) s_{j} \eta^{N-j}\right\} \geq 0
$$

Proof. (a) Choose $p(z)=1-\zeta z^{k}, k=0, \ldots, N, \zeta \in U$. Then, using (11), (18) becomes

$$
\operatorname{Re} L_{N}\left(l\left(z f^{\prime} p ; f\right)\right)=\operatorname{Re}\left\{(N+2) s_{N}-(N+2+k) \zeta s_{N-k}\right\} \geq 0
$$

which becomes (20), if we set $n=N-k$. No useful information is gained if $n>N$.

(b) Choose $p(z)=(1+\eta z) /(1-\eta z)=1+2 \sum_{k=1}^{\infty} \eta^{k} z^{k}$, and then use (11). 


\section{The Schiffer boundary variation}

It was observed by Schiffer that it is possible to compose an extremal function with analytic functions which are univalent on its range in order to ultimately produce nearby functions which serve as competitors for $f[10$, p. $433 \mathrm{ff}]$.

Generally speaking, if $f \in S$ and $H$ is analytic and univalent on $f(U)$, then $f_{H}(z)=(H(f(z))-H(0)) / H^{\prime}(0) \in S$. The invariance and composition laws for the Schwarzian derivative combine to yield the relation

$$
\left\{f_{H}, z\right\}=\{H, f(z)\}\left(f^{\prime}(z)\right)^{2}+\{f, z\}
$$

Now if $f$ is an extremal function for the problem

$$
\hat{s}_{N}=\max _{f \in S} \operatorname{Re}\left\{s_{N}(f)\right\},
$$

then we may directly conclude that

$$
\operatorname{Re} L_{N}\left(\{H, f(z)\}\left(f^{\prime}(z)\right)^{2}\right) \leq 0
$$

for any such $H$.

We consider a specific example. Let $\alpha$ and $\beta$ be distinct finite points on $\Gamma=\hat{\mathbf{C}}-f(U)$, where $f$ is an extremal function. Then some branch of $L_{\alpha, \beta}(w)=$ $\log [(w-\alpha) /(w-\beta)]$ is single-valued, analytic and univalent in $f(U)$. The functions $H_{\alpha, \beta}(w)=(\beta-\alpha) / L_{\alpha, \beta}(w)+(\alpha+\beta) / 2$ are also single-valued, analytic and univalent in $f(U)$, so that the composed functions defined by $f_{\alpha, \beta}(z)=$ $\left(H_{\alpha, \beta}(f(z))-H_{\alpha, \beta}(0)\right) / H_{\alpha, \beta}^{\prime}(0)$ belong to $S$. Consequently, we deduce

$$
\operatorname{Re} L_{N}\left(\left\{L_{\alpha, \beta}, f(z)\right\}\left(f^{\prime}(z)\right)^{2}\right) \leq 0
$$

equivalently, since

$$
\left\{L_{\alpha, \beta}, w\right\}=\frac{(\beta-\alpha)^{2}}{2(w-\alpha)^{2}(w-\beta)^{2}}
$$

we may write

$$
\operatorname{Re} L_{N}\left(\frac{(\beta-\alpha)^{2}\left(f^{\prime}(z)\right)^{2}}{(f(z)-\alpha)^{2}(f(z)-\beta)^{2}}\right) \leq 0
$$

for every choice of $\alpha, \beta \in \Gamma$. This inequality may be written in a more explicit form after evaluation by the functional $L_{N}$. To do this, we recall the following useful concept. 
For $f(z)=z+a_{2} z^{2}+\cdots \in S$, the functions $F_{m}(t)$ generated by

$$
\log \left(\frac{f(z)}{z(1-t f(z))}\right)=\sum_{m=1}^{\infty} \frac{1}{m} F_{m}(t) z^{m}
$$

for $z$ belonging to a neighborhood of the origin (depending on $t$ ) are called the Faber polynomials of $f[11$, p. 40]. Differentiation with respect to $t$ yields

$$
\frac{f(z)}{1-t f(z)}=\sum_{m=1}^{\infty} \frac{1}{m} F_{m}^{\prime}(t) z^{m} .
$$

Note that if $t=0$, then $f(z)=\sum_{m=1}^{\infty}(1 / m) F_{m}^{\prime}(0) z^{m}$, so that $m a_{m}=F_{m}^{\prime}(0)$ for every $m=1,2, \ldots$ Differentiation of (25) with respect to $z$ gives

$$
\frac{f^{\prime}(z)}{(1-t f(z))^{2}}=\sum_{m=0}^{\infty} F_{m+1}^{\prime}(t) z^{m} .
$$

Consequently, by setting $t=1 / \alpha$ and $1 / \beta$ in (26), inequality (23) becomes

$$
\operatorname{Re}\left\{\left(\frac{\beta-\alpha}{\alpha \beta}\right)^{2} \sum_{\substack{m+k=N \\ m, k \geq 0}} F_{m+1}^{\prime}(1 / \alpha) F_{k+1}^{\prime}(1 / \beta)\right\} \leq 0
$$

for all $\alpha, \beta \in \Gamma$. Furthermore, as $\beta \rightarrow \infty$, we obtain the special relation

$$
\operatorname{Re}\left\{\frac{1}{\alpha^{2}} \sum_{\substack{m+k=N \\ m, k \geq 0}}(k+1) a_{k+1} F_{m+1}^{\prime}(1 / \alpha)\right\} \leq 0
$$

for every $\alpha \in \Gamma$. These inequalities provide implicit restrictions on the omitted set $\Gamma$. They imply that the image of $\Gamma$ under a certain rational function lies in the closed left half plane.

We now consider a method of varying Schwarzian coefficients which follows from the discussion above. It was Schiffer's observation [10, p. 434] that, whenever $\alpha, \beta \in \Gamma$ are close to one another, it is possible to produce variations of functions of interest. He used these variations to show that an extremal function must satisfy a certain quadratic differential equation and that $\partial f(U)$ must consist of a finite union of analytic arcs.

Near $\infty, L_{\alpha, \beta}(w)$ has an expansion of the form

$$
L_{\alpha, \beta}(w)=-\log \left\{1-\frac{\beta-\alpha}{w-\alpha}\right\}=\sum_{k=1}^{\infty} \frac{1}{k}\left(\frac{\beta-\alpha}{w-\alpha}\right)^{k}
$$


in powers of $(w-\alpha)^{-1}$. Consequently, if $\alpha, \beta \in \Gamma$ and $|\beta-\alpha|=\varrho$ is small, then

$$
H_{\alpha, \beta}(w)=w-\frac{(\beta-\alpha)^{2}}{12(w-\alpha)}-\frac{(\beta-\alpha)^{3}}{24(w-\alpha)^{2}}+O\left(\varrho^{4}\right)
$$

where $O\left(\varrho^{4}\right)$ denotes an error term which is bounded by a constant multiple of $\varrho^{4}$ as $\varrho \rightarrow 0$, uniformly in each set $|w-\alpha| \geq \varepsilon>0$. If $f \in S$ and $\alpha, \beta \in \Gamma=\hat{\mathbf{C}}-f(U)$, then by composing $f$ with $H_{\alpha, \beta}$ and normalizing, we obtain

$$
\begin{aligned}
f_{\alpha, \beta}(z)= & f(z)-(\beta-\alpha)^{2}\left(\frac{f^{2}(z)}{12 \alpha^{2}(f(z)-\alpha)}\right) \\
& +(\beta-\alpha)^{3}\left(\frac{f^{2}(z)(2 f(z)-3 \alpha)}{24 \alpha^{3}(f(z)-\alpha)^{2}}\right)+O\left(\varrho^{4}\right)
\end{aligned}
$$

which belongs to $S$. In view of the Variational Lemma, we obtain

$$
\begin{aligned}
s_{N}\left(f_{\alpha, \beta}\right)= & s_{N}(f)-\frac{(\beta-\alpha)^{2}}{12 \alpha^{2}} L_{N}\left(l\left(\frac{f^{2}}{f-\alpha} ; f\right)\right) \\
& +\frac{(\beta-\alpha)^{3}}{24 \alpha^{3}} L_{N}\left(l\left(\frac{f^{2}(2 f-3 \alpha)}{(f-\alpha)^{2}} ; f\right)\right)+O\left(\varrho^{4}\right) \\
= & s_{N}(f)+\frac{1}{2}(\beta-\alpha)^{2} Q_{N}(\alpha ; f)+(\beta-\alpha)^{3} R_{N}(\alpha ; f)+O\left(\varrho^{4}\right)
\end{aligned}
$$

where

$$
Q_{N}(\alpha ; f)=L_{N}\left(\frac{f^{\prime}(z)^{2}}{(f(z)-\alpha)^{4}}\right)=\sum_{n=0}^{N} \frac{1}{\alpha^{n+4}}\left(\begin{array}{c}
n+3 \\
3
\end{array}\right) L_{N}\left(f^{n}\left(f^{\prime}\right)^{2}\right)
$$

and

$$
R_{N}(\alpha ; f)=L_{N}\left(\frac{f^{\prime}(z)^{2}}{(f(z)-\alpha)^{5}}\right)=-\sum_{n=0}^{N} \frac{1}{\alpha^{n+5}}\left(\begin{array}{c}
n+4 \\
4
\end{array}\right) L_{N}\left(f^{n}\left(f^{\prime}\right)^{2}\right)
$$

from which it is clear that

$$
R_{N}(\alpha ; f)=\frac{1}{4} \frac{d}{d \alpha} Q_{N}(\alpha ; f)
$$

Evidently, $Q_{N}$ is a polynomial of degree $N+4$ in $1 / \alpha$, whose coefficients may be written in terms of the coefficients of $f$. Indeed, if we write $f^{n}(z)=\sum_{k=n}^{\infty} a_{k}^{(n)} z^{k}=$ $\sum_{k=0}^{\infty} a_{k+n}^{(n)} z^{k+n}$, then for $n \leq N$, we get

$$
\begin{aligned}
L_{N}\left(f^{n} \cdot\left(f^{\prime}\right)^{2}\right) & =\frac{1}{n+1} L_{N}\left(\left(f^{n+1}\right)^{\prime} \cdot f^{\prime}\right) \\
& =\frac{1}{n+1} \sum_{m+k+n=N}(m+1)(n+k+1) a_{n+k+1}^{(n+1)} a_{m+1} .
\end{aligned}
$$


An alternate expression for $Q_{N}$ will be extremely useful. From (26) and (31), we deduce that

$$
Q_{N}(\alpha ; f)=\frac{1}{\alpha^{4}} \sum_{\substack{m+k=N \\ m, k \geq 0}} F_{m+1}^{\prime}(1 / \alpha) F_{k+1}^{\prime}(1 / \alpha)
$$

where $F_{m}$ denotes the $m$-th Faber polynomial of $f$.

Clearly, $Q_{N}$ is defined for values of $\alpha=f(z), z \in \partial U$. We wish to extend this definition to $U-\{0\}$, and to consider in depth the extended function. To do this, we must first recall a definition.

The Grunsky coefficients $c_{n k}$ of a function $f \in S$ are the coefficients generated from $f$ by setting

$$
\Phi(z, \zeta)=\log \left(\frac{f(z)-f(\zeta)}{z-\zeta}\right)=+\sum_{n=0}^{\infty} \sum_{k=0}^{\infty} c_{n k} z^{n} \zeta^{k} .
$$

Note that $\Phi$ is analytic in $U \times U$ since $f$ is univalent in $U[11$, p. 46]. A tedious computation shows that

$$
\{f, z\}=6 \cdot \lim _{\zeta \rightarrow z} \frac{\partial^{2} \Phi(z, \zeta)}{\partial z \partial \zeta} .
$$

By substituting (36) into (37) and comparing the result with (2), we see that

$$
s_{N}=6 \sum_{\substack{k+m=N+2 \\ k, m \geq 1}} k m c_{k m} .
$$

Thus, each Schwarzian coefficient is a "slanted" linear combination of Grunsky coefficients. The Grunsky coefficients are also related to the Faber polynomials. It is well known that for each $m=1,2, \ldots$, the relation

$$
F_{m}(1 / f(z))=z^{-m}-m \sum_{n=1}^{\infty} c_{m n} z^{n}
$$

holds for all $z \in U-\{0\}$ [11, p. 41].

We are now ready to prove an important

Lemma. Let $f \in S$. For $z \in U-\{0\}$, define

$$
\begin{aligned}
G_{N}(z) & =6 \sum_{m+n=N+2}\left[z \frac{d}{d z} F_{n}(1 / f(z))\right]\left[z \frac{d}{d z} F_{m}(1 / f(z))\right] \\
& =\sum_{p=-N-2}^{\infty} g_{p} z^{p}
\end{aligned}
$$


where $F_{m}$ denotes the $m$-th Faber polynomial of $f$. Then,

$$
\begin{aligned}
g_{-N-2} & =(N+1)(N+2)(N+3) \\
g_{-N-1} & =0 \\
g_{-p} & =(N+2+p) s_{N-p} \\
g_{+1} & =(N+1) s_{N+1}
\end{aligned}
$$$$
(p=0, \ldots, N)
$$

and, for $p \geq 2$,

$$
g_{+p}=12 \sum_{n+m=N+p+2}^{*}(m-p) n m c_{n m}+6 \sum_{m+n=N+2} \sum_{k+l=p} m n k l c_{m k} c_{n l}
$$

where $\sum^{*}$ denotes a sum taken over $m$ and $n$ such that $n+m=N+p+2$, with the restrictions that $p+1 \leq m \leq N+p+1$ and $1 \leq n \leq N+1$.

Moreover, if $G_{N}(z)$ is real on $|z|=1$ and continuous on $\bar{U}-\{0\}$, then $\overline{G_{N}(z)}=G_{N}(1 / \bar{z})$ for $0<|z|<\infty$, and $G_{N}$ assumes the canonical form

$$
G_{N}(z)=g_{0}+\sum_{p=1}^{N+2}\left(\bar{g}_{+p} z^{-p}+g_{+p} z^{p}\right)
$$

Proof. The coefficients $g_{p}$ of $G_{N}$ are determined by substituting (39) into (40), and equating coefficients while making use of (38). The sum $\sum^{*}$ may be described as an "incomplete, weighted, slanted sum", and the double sum as a "double convolution product" of a submatrix of the Grunsky matrix with itself.

Due to relation (39), it is clear that $G_{N}$ has a pole of order $N+2$ at the origin, and no other poles in $U-\{0\}$. By the Koebe $\frac{1}{4}$-Theorem [3, p. 31], $|1 / f(z)| \leq 4$ for $z \in \partial U$, so that $G_{N}$ has no poles on $\partial U$. Now, if $G_{N}$ is continuous on $\bar{U}-\{0\}$ and is real on $\partial U$, then the Schwarz Reflection Principle allows us to conclude that $G_{N}$ has an extension to $0<|z|<+\infty$ which satisfies $\overline{G_{N}(z)}=G_{N}(1 / \bar{z})$, which forces $G_{N}$ to have the form (42). Note, in particular, that $g_{0}$ is real, that the coefficients $g_{-p}$ and $g_{+p}$ are symmetric with respect to the real axis and that $g_{+p}=0$ for all $p \geq N+3$. ㅁ

We may now prove

Theorem 1. Let $f \in S$ be extremal for the problem

$$
\hat{s}_{N}=\max _{f \in S} \operatorname{Re}\left\{s_{N}\right\} .
$$

Then, $f$ satisfies the quadratic differential equation

$$
\begin{gathered}
6 \sum_{m+n=N+2}\left[z \frac{d}{d z} F_{m}(1 / f(z))\right]\left[z \frac{d}{d z} F_{n}(1 / f(z))\right] \\
=(N+2) s_{N}+\sum_{p=1}^{N}(N+2+p)\left(s_{N-p} z^{-p}+\overline{s_{N-p}} z^{+p}\right) \\
+(N+1)(N+2)(N+3)\left(z^{-N-2}+z^{+N+2}\right)
\end{gathered}
$$


where $F_{m}$ denotes the $m$-th Faber polynomial of $f$ and $s_{m}$ denote the Schwarzian coefficients of $f$. Also, the set $\Gamma_{f}$ omitted by $f$ consists of a finite union of analytic arcs.

Proof. In his dissertation, Overholt [8, p. 39] considered the problem

$$
\max _{h \in S} \operatorname{Re} L(\{h, z\})
$$

where $L$ is a continuous linear functional on $H(U)$, the class of analytic functions on the unit disk. He used Schiffer's Theorem [3, p. 297] to show that if $f$ is an extremal function for this problem, then its omitted set $\Gamma_{f}$ consists of finitely many analytic trajectory arcs of the quadratic differential

$$
-L\left(\frac{\left(f^{\prime}(z)\right)^{2}}{(f(z)-w)^{4}}\right) d w^{2} .
$$

With $L=L_{N}$, this conclusion is equivalent to the statement that $\Gamma_{f}$ is the finite union of analytic arcs parametrized by $w=w(t)$ satisfying the quadratic differential equation

$$
Q_{N}(w) d w^{2}=-\frac{1}{w^{4}} \sum_{m+k=N} F_{m+1}^{\prime}(1 / w) F_{k+1}^{\prime}(1 / w) d w^{2}>0 .
$$

This differential equation for the omitted arc leads naturally to a differential equation for the extremal function. Since $\Gamma_{f}$ is the finite union of analytic arcs, $f$ has an analytic continuation across the unit circle, except at finitely many points. Also, $\Gamma_{f}$ has the parametrization $w(t)=f\left(e^{i t}\right), w^{\prime}(t)=i e^{i t} f\left(e^{i t}\right)$. The differential equation (44) therefore asserts that the meromorphic function $G_{N}$ defined in the previous lemma is continuous, real and non-negative on the unit circle when $f$ is extremal. Thus, $G_{N}$ has the form (42) and its coefficients are given by (41), all of which results in equation (43). Unfortunately, this equation involves the initial coefficients of the unknown extremal function. We observe in passing that the fact that $g_{0}=(N+2) s_{N}$ is real could also have been obtained through the use of the rotation variation $(f \rightarrow \bar{\eta} f(\eta z))$. Also, the symmetric coefficient condition $g_{1}=\overline{g_{-1}}$ may be rewritten as

$$
(N+1) s_{N+1}=(N+3) \overline{s_{N-1}}
$$

and could have otherwise been obtained through the use of the Marty Variation $\left[3\right.$, p. 59]. This suggests that the necessary conditions $g_{+p}=\overline{g_{-p}}(p=2, \ldots, N)$ and $g_{+p}=0(p=N+3, \ldots)$ involving the Schwarzian and Grunsky coefficients of the extremal function, and hence the differential equation (43) itself, may be viewed as an infinite succession of "higher Marty relations". We also wish to point out here that Proposition 1(a), obtained as an application of the spire variation, may now be interpreted to say that $\left|g_{ \pm p}\right| \leq g_{0}(p=0, \ldots, N)$, while Proposition 1(b) confirms the conclusion that $G_{N}$ is non-negative on the unit circle. $\square$ 
Our next goal is to understand in greater depth the geometry of the extremal configuration; i.e., to describe the omitted set $\Gamma_{f}=\hat{\mathbf{C}}-f(U)$. We wish to show that the quadratic differential $Q_{N}(w) d w^{2}$ can have only simple zeroes on $\Gamma_{f}$. The general theory of the trajectory structure of quadratic differentials $[9$, Chapter 8] will then imply that the omitted analytic arcs may branch out in at most three equiangular directions at each occurring zero. The required arguments may be carried out within the established framework with respect to the class $S$, or equivalently, with respect to the class $\Sigma$. For technical reasons we now transfer our arguments to $\Sigma$.

As it has been pointed out, if $f \in S$ is extremal for the coefficient problem $\max _{f \in S} \operatorname{Re}\left\{s_{N}(f)\right\}$, then $g(z)=1 / f(1 / z)$ belongs to $\Sigma, s_{N}(f)=\sigma_{N}(g)$ and $g$ is extremal for the problem $\max _{g \in \Sigma} \operatorname{Re}\left\{\sigma_{N}(g)\right\}$. The omitted set $\Gamma_{g}=\hat{\mathbf{C}}-g(\Delta)$ for an extremal $g \in \Sigma$ may be obtained by inverting the omitted set $\Gamma_{f}=\hat{\mathbf{C}}-f(U)$ of an extremal $f \in S$; i.e., if $w \in \Gamma_{f}$, then $v \in \Gamma_{g}$, where $v=1 / w$. Indeed, since $d v^{2}=d w^{2} / w^{4}$ and

$$
L_{N}\left(f^{n}\left(f^{\prime}\right)^{2}\right)=L_{-N-4}\left(g^{2} / g^{n+4}\right),
$$

the omitted set $\Gamma_{g}$ consists of finitely many analytic trajectory arcs of the quadratic differential

$$
P_{N}(v) d v^{2}=-\left(\sum_{n=0}^{N}\left(\begin{array}{c}
n+3 \\
3
\end{array}\right) L_{-N-4}\left(g^{\prime 2} / g^{n+4}\right) v^{n}\right) d v^{2}
$$

which is obtained in this form from (31) by substitution.

We are now ready to prove

Theorem 2. The quadratic differential $P_{N}(v) d v^{2}$ can have only simple zeroes on the omitted set $\Gamma_{g}$.

Proof. Leung and Schober [6] have recently proven a general result of this type for support points in $\Sigma$. Their argument is adapted here to reflect the fact that we are dealing with the Schwarzian derivative and its coefficients.

The first phase of our argument utilizes the Schiffer truncation variation to show that the presence of a $k$-th order zero on $\Gamma_{g}$ implies that the Schwarzian coefficient inequality $\operatorname{Re}\left\{\sigma_{k}(G)\right\} \geq 0$ is valid for all $G(w)=w+\sum_{j=0}^{\infty} c_{j} w^{-j}$ which are analytic and univalent in the complement of the linear segment $[0,4]$. Let us denote this class by $\mathscr{G}$. We then show that there exist functions $G_{k} \in \mathscr{G}$ for which $\operatorname{Re}\left\{\sigma_{k}\left(G_{k}\right)\right\}<0$ for each $k \geq 2$, thus eliminating the possibility of zeroes on $\Gamma_{g}$ of multiplicity greater than one.

After a translation, we may assume that $v=0$ is a zero of order $k$ for $P_{N}(v)$. This condition holds if and only if $L_{-N-4}\left(g^{2} / g^{n+4}\right)=0$ for $n=0, \ldots, k-1$ and $L_{-N-4}\left(g^{\prime 2} / g^{k+4}\right) \neq 0$, due to the form of $P_{N}$. Furthermore, after rotating the functional and the function, we may also assume that $L_{-N-4}\left(g^{\prime 2} / g^{k+4}\right)<0$ and 
that one of the omitted arcs emanates from the origin in the positive horizontal direction (i.e., $P_{N}(v) d v^{2} \approx\left(c v^{k}+\ldots\right) d v^{2}$ near $v=0$ with $c>0$ ).

To apply Schiffer's truncation [2, p. 216], we consider a disk $|v| \leq \delta$ for a sufficiently small positive $\delta$. We delete all arcs of $\Gamma_{g}$ except for that connected part of the arc emanating from the origin in the positive horizontal direction that lies inside this disk. Designate this subarc by $\Gamma_{g}^{\delta}$, and let $F_{\delta}$ be the conformal map from $\Delta$ onto the complement of $\Gamma_{g}^{\delta}$ with an expansion of the form $F_{\delta}(z)=\varrho z+0(1)$ around $z=\infty$ with $\varrho>0$. Then $F_{\delta}$ and the original extremal $g$ are related by a Schwarz function $w: \Delta \rightarrow \Delta$ satisfying $F_{\delta} \circ w=g$ and $w^{\prime}(\infty)=1 / p$. As $\delta \rightarrow 0$, we have $\varrho \rightarrow 0$. The function $g_{\delta}=(1 / \varrho) F_{\delta}$ in $\Sigma$ is extremal for the new functional $\operatorname{Re} \lambda_{\delta}(g)$, defined by $\lambda_{\delta}(g)=\sigma_{N}(\varrho g \circ w)$, since $\lambda_{\delta}\left(g_{\delta}\right)=\sigma_{N}\left(F_{\delta} \circ w\right)=\sigma_{N}(g)$. Let us designate the set omitted by $g_{\delta}$ to be $\gamma_{g}^{\delta}$, obtained from $\Gamma_{g}^{\delta}$ by dilating by the factor $1 / \varrho$. Since $\gamma_{g}^{\delta}$ is an arc emanating from $w=0$ in the positive horizontal direction, it approaches the line segment $[0,4]$ as $\delta \rightarrow 0$. By the Carathéodory Convergence Theorem, $g_{\delta}$ approaches the Koebe function $K(z)=z+2+1 / z$ as $\delta \rightarrow 0$.

Now let $G_{\delta}$ be analytic and univalent in the complement of $\gamma_{g}^{\delta}$ and have an expansion of the form $G_{\delta}(w)=w+\sum_{j=0}^{\infty} c_{j}(\delta) w^{-j}$ around $w=\infty$. Then, $g_{\delta}^{*}=G_{\delta} \circ g_{\delta}$ serves as a variation of $g_{\delta}$, and so $\operatorname{Re} \lambda_{\delta}\left(g_{\delta}^{*}\right) \leq \operatorname{Re} \lambda_{\delta}\left(g_{\delta}\right)$, or $\operatorname{Re} \sigma_{N}\left(g_{\delta}^{*} \circ w\right) \leq \operatorname{Re} \sigma_{N}\left(g_{\delta} \circ w\right)$, by using the definition of $\lambda_{\delta}$. Since $g_{\delta}^{*} \circ w=$ $G_{\delta} \circ g_{\delta} \circ w$, we conclude that $\operatorname{Re} L_{-N-4}\left(\left\{G_{\delta} \circ g_{\delta} \circ w, z\right\}\right) \leq \operatorname{Re} L_{-N-4}\left(\left\{g_{\delta} \circ w, z\right\}\right)$, or $\operatorname{Re} L_{-N-4}\left(\left\{G_{\delta}, g_{\delta} \circ w\right\}\left(g_{\delta} \circ w\right)^{\prime 2}\right) \leq 0$, due to the Schwarzian composition law (6). Since $g_{\delta} \circ w(z)=g(z) / \varrho$, this last condition becomes

$$
\operatorname{Re} L_{-N-4}\left(\left\{G_{\delta}, g(z) / \varrho\right\}\left(g^{\prime}(z)\right)^{2}\right) \leq 0,
$$

which may be interpreted as a coefficient inequality. For if we write $\left\{G_{\delta}, w\right\}=$ $\sum_{n=0}^{\infty} \sigma_{n}\left(G_{\delta}\right) w^{-n-4}$, this condition becomes

$$
\operatorname{Re} L_{-N-4}\left(\sum_{n=0}^{\infty} \sigma_{n}\left(G_{\delta}\right) \varrho^{n+4}\left(g^{\prime}(z)\right)^{2} /(g(z))^{n+4}\right) \leq 0 .
$$

Our assumption on the order of the zero allows us to write this inequality in the simplified form

$$
\operatorname{Re}\left\{\sigma_{k}\left(G_{\delta}\right) \varrho^{k+4} L_{-N-4}\left(g^{\prime 2} / g^{k+4}\right)+0\left(\varrho^{k+5}\right)\right\} \leq 0 .
$$

Recalling that $L_{-N-4}\left(g^{\prime 2} / g^{k+4}\right)<0$, dividing by $\varrho^{k+4}$ and letting $\varrho \rightarrow 0(\delta \rightarrow 0)$, leads us to the conclusion that $\operatorname{Re} \sigma_{k}(G) \geq 0$ for all $G \in \mathscr{G}$.

In the next phase of our argument, we produce a partial differential equation for Löwner chains in $\mathscr{G}$, and then use a second order variation of the identity in $\mathscr{G}$ 
to produce functions $G_{k} \in \mathscr{G}$ such that $\operatorname{Re}\left\{\sigma_{k}\left(G_{k}\right)\right\}<0$ for all $k \geq 2$, thereby eliminating all zeroes of order $k \geq 2$, from the omitted set.

We begin with the familiar Löwner equation for the class $S$. Let $\mathscr{K}=\mathscr{K}(t)$ be a continuous complex-valued function on $[0, \infty)$ with $|\mathscr{K}(t)|=1$. Then the differential equation

$$
\frac{\partial f}{\partial t}=z\left(\frac{1+\mathscr{K} z}{1-\mathscr{K} z}\right) \frac{\partial f}{\partial z} \quad(t \geq 0, z \in U)
$$

has a solution such that the functions $e^{-t} f(z, t) \in S, t \geq 0$. In order to refer the Löwner chain $f(z, t)$ to the complement of the segment $[0,4]$, we make use of the function $K(z)=z+2+1 / z$, which maps $U \backslash\{0\}$ onto the complement of $[0,4]$. Denote the inverse of $K$ by $S(w)=(w-2-w \sqrt{1-4 / w}) / 2$, where the branch of the square root is chosen so that $\sqrt{1}=1$. The corresponding Löwner chain of mappings $F(\cdot, t)$ defined in the complement of $[0,4]$ are given by the composition $F(w, t)=1 / f(S(w), t)$. From (46) and the chain rule, one finds that $F(w, t)$ satisfies the differential equation

$$
\frac{\partial F}{\partial t}=R(w, t) \frac{\partial F}{\partial w} \quad(t \geq 0)
$$

where

$$
R(w, t)=\left(S(w)-\frac{1}{S(w)}\right)\left(\frac{1+\mathscr{K}(t) S(w)}{1-\mathscr{K}(t) S(w)}\right)
$$

and that $G(w, t)=e^{t} F(w, t) \in \mathscr{G}$. We use (47) to produce a Löwner equation for the chain $\{G(w, t), w\}$ of Schwarzians. To do this, replace $w$ by $v$ in (47), and subtract the resulting equations. Multiply the result by $e^{t}$, divide by $G(w, t)-$ $G(v, t)$, and insert $\log (w-v)$ where necessary to obtain

$$
-1+\frac{\partial \Phi}{\partial t}=R(w, t) \frac{\partial \Phi}{\partial w}+R(v, t) \frac{\partial \Phi}{\partial v}+\frac{R(w, t)-R(v, t)}{w-v}
$$

where

$$
\Phi(w, v, t)=\log \left(\frac{G(w, t)-G(v, t)}{w-v}\right) .
$$

Now since the Schwarzian may also be defined in the class $\mathscr{G}$ by

$$
\{G(w, t), w\}=6 \lim _{v \rightarrow w} \Phi_{w v}(w, v, t),
$$

we may differentiate (49) and let $v \rightarrow w$ to obtain the differential equation

$$
\begin{aligned}
\frac{\partial}{\partial t}\{G(w, t), w\}= & R(w, t) \frac{\partial}{\partial w}\{G(w, t), w\} \\
& +2\{G(w, t), w\} \frac{\partial}{\partial w} R(w, t)+\frac{\partial^{3}}{\partial w^{3}} R(w, t) .
\end{aligned}
$$


The Schwarzian coefficients of a function $G \in \mathscr{G}$ are defined by the relation

$$
\{G, w\}=\sum_{k=0}^{\infty} \sigma_{k}(G) w^{-k-4}
$$

We will use (50) and a variational argument to show that there exist functions $G_{k} \in \mathscr{G}$ such that $\operatorname{Re}\left\{\sigma_{k}\left(G_{k}\right)\right\}<0$ for all $k \geq 2$, but first we wish to remark that $\operatorname{Re}\left\{\sigma_{k}(G)\right\} \geq 0$ for all $G \in \mathscr{G}$, if $k=0,1$. If $g(z)=z+b_{0}+b_{1} / z+\cdots \in \Sigma$, then $G(w)=g(1 / S(w)) \in \mathscr{G}$, so that $G(w)=w+\left(b_{0}-2\right)+\left(b_{1}-1\right) w^{-1}+$ $\left(b_{2}+2 b_{1}-2\right) w^{-2}+\left(b_{3}+4 b_{2}+5 b_{1}-5\right) w^{-3}+\cdots$ and every $G \in \mathscr{G}$ may be written in this manner. An elementary computation shows that

$$
\begin{aligned}
& \sigma_{0}(G)=6\left(1-b_{1}\right) \\
& \sigma_{1}(G)=24\left(2-2 b_{1}-b_{2}\right) \\
& \sigma_{2}(G)=12\left(24-5 b_{3}-20 b_{2}-23 b_{1}-b_{1}^{2}\right)
\end{aligned}
$$

and so forth. It is well known $\left[3\right.$, p. 134] that $\operatorname{Re}\left\{b_{1}\right\} \leq 1$, so that $\operatorname{Re} \sigma_{0}(G) \geq 0$ for all $G \in \mathscr{G}$. Also, Garabedian and Schiffer [4] have shown that $\operatorname{Re}\left\{2 b_{1}+b_{2}\right\} \leq 2$, implying that $\operatorname{Re}\left\{\sigma_{1}(G)\right\} \geq 0$ for all $G \in \mathscr{G}$. The statement that there exists a $G \in \mathscr{G}$ for which $\operatorname{Re}\left\{\sigma_{2}(G)\right\}<0$ may be interpreted to mean that there exists a $g \in \Sigma$ for which $\operatorname{Re}\left\{5 b_{3}+20 b_{2}+23 b_{1}+b_{1}^{2}\right\}>24$, and so forth, for $k \geq 3$.

Choose $\mathscr{K}(t)=e^{i \varepsilon \Theta(t)}$, where $\varepsilon$ is a small real parameter and $\Theta$ is a bounded continuous function of $t$. Then, we have the asymptotic expansions

$$
R(w, t, \varepsilon)=-w-\varepsilon \frac{2 i \Theta(t)}{\sqrt{1-4 / w}}+\varepsilon^{2} \frac{\Theta^{2}(t)}{(1-4 / w)}+o\left(\varepsilon^{2}\right)
$$

and

$$
\{G(w, t, \varepsilon), w\}=\phi(w, t)+\varepsilon V(w, t)+\varepsilon^{2} Q(w, t)+o\left(\varepsilon^{2}\right)
$$

as $\varepsilon \rightarrow 0$. If $\varepsilon=0$, then $\mathscr{K}(t) \equiv 1$, independent of $t$, and the solution to (46) is $f(z, t)=e^{t} z /(1+z)^{2}$, so that $G(w, t)=w$. Hence $\phi(w, t) \equiv 0$. Substituting these expansions into (49) and equating coefficients of $\varepsilon$, we obtain the equations

$$
\frac{\partial V}{\partial t}=-w \frac{\partial}{\partial w} V(w, t)-2 V(w, t)-2 i \Theta(t)\left(\frac{1}{\sqrt{1-4 / w}}\right)^{\prime \prime \prime}
$$

and

$$
\frac{\partial Q}{\partial t}=-w \frac{\partial}{\partial w} Q(w, t)-2 Q(w, t)-2 i \Theta(t) \frac{\partial}{\partial w}\left(\frac{V(w, t)}{\sqrt{1-4 / w}}\right)+\Theta^{2}(t)\left(\frac{1}{1-4 / w}\right)^{\prime \prime \prime}
$$


Since $\{G(w, t, \varepsilon), w\}=O(1)$ as $w \rightarrow \infty$, the expansions of $V$ and $Q$ around infinity are of the form $V(w, t)=\sum_{n=0}^{\infty} V_{n}(t) w^{-n-4}$ and $Q(w, t)=$ $\sum_{n=0}^{\infty} Q_{n}(t) w^{-n-4}$. It follows from this system of differential equations that

$$
V_{n}^{\prime}(t)=(n+2) V_{n}(t)+2 i \Theta(t)(n+1)_{3} d_{n+1} \quad(n \geq 0)
$$

and that

$Q_{n}^{\prime}(t)=(n+2) Q_{n}(t)+2 i(n+3) \sum_{j=0}^{n-1} d_{n-1-j} V_{j}(t) \Theta(t)-4^{n+1}(n+1)_{3} \Theta^{2}(t) \quad(n \geq 1)$

where

$$
\frac{1}{\sqrt{1-4 / w}}=\sum_{n=0}^{\infty} d_{n} w^{-n}
$$

with $d_{n}=(2 n) ! /(n !)^{2}$.

If $w$ is restricted to a large disk, then $G(w, t, \varepsilon)$ is uniformly bounded. Therefore, the coefficients $V_{n}(t)$ and $Q_{n}(t)$ vanish at $t=\infty$. Thus, the system can be integrated to yield

$$
V_{n}(0)=-2 i(n+1)_{3} d_{n+1} \int_{0}^{\infty} \Theta(t) e^{-(n+2) t} d t
$$

and

$$
Q_{n}(0)=-4(n+3) \sum_{j=0}^{n-1}(j+1)_{3} d_{n-1-j} d_{j+1} \int_{0}^{\infty} \int_{t}^{\infty} \Theta(s) \Theta(t) e^{-(n-j) t-(j+2) s} d s d t
$$

$$
+4^{n+1}(n+1)_{3} \int_{0}^{\infty} \Theta^{2}(t) e^{-(n+2) t} d t .
$$

In the expansion of $\{G(w, 0, \varepsilon), w\}=\Sigma_{n=0}^{\infty} \sigma_{n}(G(0, \varepsilon)) w^{-n-4}$, the coefficients are of the form $\sigma_{n}(G(0, \varepsilon))=\varepsilon V_{n}(0)+\varepsilon^{2} Q_{n}(0)+0\left(\varepsilon^{2}\right)$. Evidently, $V_{n}(0)$ is purely imaginary and $Q_{n}(0)$ is real, so $\operatorname{Re}\left\{\sigma_{n}(G(0, \varepsilon))\right\}=\varepsilon^{2} Q_{n}(0)+O\left(\varepsilon^{3}\right)$.

Now, assume that $k \geq 2$. Substitute $\Theta(t)=e^{(2-\delta) t}(\delta>0)$ into (51), and discard all terms except the one for which $j=0$, to obtain

$$
Q_{k}(0) \leq \frac{1}{k-2+2 \delta}\left(4^{k+1}(k+1)_{3}-\frac{24(k+3) d_{k-1} d_{1}}{\delta}\right) .
$$

As $\delta \rightarrow 0$, the expression on the right becomes negative, showing that $Q_{k}(0)<0$. The function $\Theta(t)$ is not bounded, but the truncation $\Theta_{M}(t)=\min \{M, \Theta(t)\}$ is 
bounded, and an application of the Dominated Convergence Theorem leads us to conclude that $Q_{k}(0)$, and hence $\operatorname{Re}\left\{\sigma_{k}\left(G_{k}(0, \varepsilon)\right)\right\}$, is negative for some function $G_{k} \in \mathscr{G}$ determined from $\Theta_{M}(t)$.

To summarize our results, we recall that the presence of a $k$-th order zero of $P_{N}(v)$ on $\Gamma_{g}$ implied that $\operatorname{Re}\left\{\sigma_{k}(G)\right\} \geq 0$ for every $G \in \mathscr{G} ;$ since we have shown that there exists functions $G_{k}(w, 0, \varepsilon) \in \mathscr{G}$ for which $\operatorname{Re}\left\{\sigma_{k}\left(G_{k}(0, \varepsilon)\right)\right\}<0$, for every $k \geq 2$ we have eliminated the presence of $k$-th order zeroes for each $k \geq 2$. $\square$

Corollary 2.1. The functions $K_{m}(z)=\left(K\left(z^{m}\right)\right)^{1 / m}, m \geq 4$, where $K(z)=$ $z+2+1 / z$, cannot be solutions to the problem

$$
\hat{\sigma}_{N}=\max _{g \in \Sigma} \operatorname{Re}\left\{\sigma_{N}(g)\right\}
$$

for any $N \geq 2$. Consequently, $\hat{\sigma}_{N}>\sigma_{N}\left(K_{N+2}\right)=2(N+1)(N+3)$, for $N \geq 2$.

Proof. The functions $K_{m}(z)$ map $\Delta$ onto the plane less $m$ symmetric, equally spaced radial slits emanating from the origin. If $K_{m}$ were extremal, then these slits would lie on the trajectories of the quadratic differential $P_{N}(v) d v^{2}$, and would imply the existence of a zero of order $m-2$ at the origin. But this has been shown to be impossible if $m-2 \geq 2$. $\square$

We close this paper with a short discussion of the extremal functions in $S$ when $N=0,1,2$. The cases $N \geq 3$ will be discussed in a later paper.

In $N=0$, then $Q_{0}(w) d w^{2}=-d w^{2} / w^{4}$. Consequently, the omitted set lies on the imaginary axis. Utilizing the Schwarz Reflection Principle in the usual way, we deduce that the extremal function satisfies the equation

$$
\left(\frac{z f^{\prime}(z)}{f^{2}(z)}\right)^{2}=\frac{(z+i)^{2}(z-i)^{2}}{z^{2}}
$$

which is easily integrated to obtain $f(z)=z /(1 \pm i z)^{2}$, a rotated Koebe function.

If $N=1$, the author has shown [12, Theorem 1] by other methods that the extremal function is $f(z)=z /\left(1-z^{3}\right)^{2 / 3}$. With $a_{2}=0$, the quadratic differential reduces to $Q_{1}(w) d w^{2}=-4 d w^{2} / w^{5}$, and the corresponding differential equation is

$$
\frac{z^{2} f^{\prime 2}(z)}{f^{5}(z)}=\frac{(z+1)^{2}\left(z-e^{i \pi / 3}\right)^{2}\left(z-e^{-i \pi / 3}\right)^{2}}{z^{3}} .
$$

The omitted set consists of three equally spaced, symmetric, radial arcs parametrized by $w(t)=c t, c=-1, e^{i \pi / 3}, e^{-i \pi / 3}$ and $t \in\left[2^{-2 / 3}, \infty\right)$.

If $N=2$, then the author has previously shown by other methods [12, Theorem 2] that the extremal function $f(z)$ in this case is the square-root transform of the extremal function $f_{\lambda}(z)=z+a_{2}(\lambda) z^{2}+\cdots$ for the coefficient problem $\max _{f \in S} \operatorname{Re}\left\{a_{3}-\lambda a_{2}^{2}\right\}$, with $\lambda=17 / 20$. The solution to this problem is well 
known and is discussed in detail elsewhere, $[3$, p. 104-7] or $[9$, p. 165-7]. The Löwner Theory provides the formula

$$
a_{2}\left(f_{\lambda}\right)=-2 \int_{0}^{\infty} \mathscr{K}(t) e^{-t} d t
$$

where $\mathscr{K}(t)=\cos \Theta(t)+i \sin \Theta(t)$,

$$
\cos \Theta(t)= \begin{cases}e^{t-\lambda}, & 0 \leq t \leq \lambda \\ 1, & \lambda<t<+\infty\end{cases}
$$

and

$$
\sin \Theta(t)= \begin{cases}+\left[1-e^{2(t-\lambda)}\right]^{1 / 2}, & 0 \leq t<\tau \\ -\left[1-e^{2(t-\lambda)}\right]^{1 / 2}, & \tau \leq t \leq \lambda \\ 0, & \lambda<t<+\infty\end{cases}
$$

and $\tau$ is chosen so that $\int_{0}^{\infty} e^{-t} \sin \Theta(t) d t=0$.

Hence, $a_{2}\left(f_{\lambda}\right)=-2(\lambda+1) e^{-\lambda}=-\frac{37}{10} e^{-17 / 20}=-1.5814 \ldots$ Since $f(z)=$ $\left(f_{\lambda}\left(z^{2}\right)\right)^{1 / 2}$, it follows that $a_{3}(f)=\frac{1}{2} a_{2}\left(f_{\lambda}\right)=-\frac{37}{20} e^{-17 / 20}=-0.7907 \ldots$ and that $s_{0}(f)=6\left(a_{3}(f)-a_{2}^{2}(f)\right)=6 a_{3}(f)=-\frac{111}{10} e^{-17 / 20}=-4.7443 \ldots$, as $a_{2}(f)=0$ since $f$ is odd. Hence, the quadratic differential in this case reduces to the form

$$
Q_{2}(w) d w^{2}=-\left(10+s_{0} w^{2}\right) \frac{d w^{2}}{w^{6}}
$$

and the corresponding differential equation assumes the form

$$
6 z^{2}\left(10+s_{0} f^{2}(z)\right)\left(\frac{f^{\prime}(z)}{f^{3}(z)}\right)^{2}=60 z^{-4}+6 s_{0} z^{-2}+4 s_{2}+6 s_{0} z^{2}+60 z^{4},
$$

where $s_{2}=30\left(1+2 e^{-34 / 3}\right)$.

The omitted set contains both simple zeroes of $Q_{2}(w) d w^{2}$. They are located at $\pm w_{0}$, where $w_{0}=\sqrt{10 /\left(-s_{0}\right)}=+1.4518 \ldots$ Three equally spaced analytic arcs emanate from each zero. In particular, the radial segments $\left[w_{0}, \infty\right)$ and $\left(-\infty,-w_{0}\right]$ are omitted.

In view of our work here, it may be reasonable to suggest that all zeroes of $Q_{N}(w) d w^{2}$ belong to the omitted set.

Acknowledgements. This paper was written at the University of Delaware, while on sabbatical from the Pennsylvania State University. Both universities deserve recognition for their generous assistance. The author also wishes to thank Professor Ivar Stakgold for his unwavering support, Professor Y. J. Leung for enriching mathematical discussions, and Pam Haverland for her expert typing of this difficult manuscript. 


\section{References}

[1] BERnard, S.D.: Bibliography of Schlicht functions. - Courant Institute of Mathematical Sciences, New York University, 1966; Part II, ibid., 1977. (Reprinted by Mariner Publishing Co., Tampa, Florida, 1983; Part III added.)

[2] Chang, A., M. Schiffer, and G. Schober: On the second variation for univalent functions. - J. Analyse Math. 40, 1981, 203-238.

[3] Duren, P.L.: Univalent functions. - Springer-Verlag, Heidelberg-New York, 1983.

[4] Garabedian, P.R., and M. Schiffer: A coefficient inequality for schlicht functions. Ann. of Math. (2) 61, 1955, 116-136.

[5] Lehto, O.: Univalent functions and Teichmuller spaces. - Springer-Verlag, HeidelbergNew York, 1987.

[6] Leung, Y.J., and G. Schober: The simple-zero theorem for support points in $\Sigma$. - Proc. Amer. Math. Soc. 105, 1989, 603-608.

[7] Nehari, Z.: Conformal mapping. - Dover, New York, 1975.

[8] Overholt, M.: Linear problems for the Schwarzian derivative. - Doctoral Dissertation, University of Michigan, 1987.

[9] Pommerenke, Ch.: Univalent functions with a chapter on quadratic differentials by G. Jensen. - Vandenhoeck and Ruprecht, Göttingen, 1975.

[10] Schiffer, M.: A method of variation within the family of simple functions. - Proc. London Math. Soc. 44, 1938, 432-449.

[11] Schoвer, G.: Univalent functions-Selected topics. - Lecture Notes in Mathematics 478. Springer-Verlag, 1975.

[12] Zemyan, S.: On the Schwarzian coefficients of univalent functions. - Bulletin Austral. Math. Soc. (to appear). 